\title{
Some politics and statistical physics behind Sri Lankan stock market crashes
}

\author{
Janak R. Wedagedera \\ Department of Mathematics, University of Ruhuna, Matara, Sri Lanka, janak@maths.ruh.ac.lk, \\ W. Ajith Raveendra de Mel \\ Department of Mathematics, University of Ruhuna, Matara, Sri Lanka, ajith@maths.ruh.ac.lk,
}

\begin{abstract}
We investigate the mechanism of how the so called critical crashes happen in the stock market from a statistical physics point of view. We shall consider a modelling approach proposed in Johansen et al. (2000) to study the nature of a possible crash occurred in the Sri Lankan stock market in 1994 with a mentioning of the political events of the country that had been prevailing during that time which could be the key force that had driven the market towards the said crash. We shall determine the parameters that governed this crash and fit a periodic function for the actual data based on the critical phenomena in statistical mechanics.

We also propose a modelling approach via which we illustrate the critical crashes in the market are not unusual phenomena when the market is modeled as a system in statistical physics, where we employ a version of Deridda's Random Energy Model Derrida (1980), Derrida (1997) applied to the price fluctuations in the financial market.
\end{abstract}

Key words: financial derivatives, stock market, stochastic differential equations, Cayley tree models, statistical mechanics

\section{What is a financial crash ?}

A financial crash on the macroscopic level is seen as a sudden drop of the price of a derivative or stock price. Though it seems as a drop-down in price which may take place over a few hours or a few days, the events that drive a market towards a crash would have been building-up perhaps over months or years.

Paul and Baschnagel (1999) illustrated, using Dow Jones Index data that crashes are outliers which are not explained or modeled by usual statistical fluctuations occur in the probability distribution of the index. Thus, identifying and trying to model crashes in standard statistical techniques is not possible. In fact, Johansen A. (1998) and Paul and Baschnagel (1999) demonstrated how the Dow Jones Index had decreased by a certain percentage from year 1900 to year 1994 where they illustrated when the frequency of this decrease $N(D)$ is smaller than $15 \%$ (where $D$ stands for the magnitude of the decrease or draw down), the frequency could reasonably be approximated by an exponential with $N(D)=N_{0} \exp \left(-D / D_{c}\right)$. This behaviour is consistent with the price distribution so can nicely be explained using standard statistical theory. In contrary, there have been three occasions in which the Dow Jones Index had decreased more than 25\%: during World War I, in 1927 
and in 1987 which do not fit in the above explanation. This suggest the need of a critical phenomena (Dorlas (1999), Schwabl (2002), Yeomans (1992))to explain these outliers.

The use of the word critical in the above in fact refers to such analogies one observes in complex systems where such systems dynamically driven out of equilibrium. Examples are earthquakes, avalanches, crack propagation etc Johansen et al. (2000). The first proposal for a connection between crashes and critical points was made by Sornette et al. (1996).

In the first half of this paper we shall concern on to investigate if there has been similar behaviour occurred in the Sri Lankan stock market (or commonly known as the Colombo Stock Exchange) along the same lines proposed in Johansen et al. (2000) and Paul and Baschnagel (1999). Indeed, we report on, upon a careful observation and analysis on the past data (from 1994 - 2004) of the Milanka price index of the Colombo Stock Market, Sri Lanka, about a possible critical crash which we claim to have happened most probably on the $1^{\text {st }}$ March 1994.

In the second half of the paper we propose a model which has been extensively studied in the literature (Derrida (1981), Derrida (1985), Derrida (1997)) in a different context namely, in spin glasses in statistical physics, to be used to explain how the so called critical crashes could take place in financial market when we model the stock price process as spin glass or a directed polymer along a Cayley tree (Buffet et al. (1993)).

\subsection{March 1994 Crash in the Sri Lankan stock market}

In Figure 1 we show plots of data of the main financial index used in the Sri Lankan stock market, namely the Milanka Index for the period 1994-2004. We observe a crash-like behaviour on the 1st March 2004 which had resulted due to the economical and political factors that prevailed in the country during that time. In fact, on the $1^{\text {st }}$ of March 1994, the political picture of the county enters into a new regime as then governing party had a defeat at the Provincial Council Elections that had been held the following day, which had triggered a sequence of dramatic political events that ultimately lead to a change in the government as well as the Presidents office. This has been the key factor for the crash-like behavior of the market observed just before the date of the election.

In Johansen et al. (2000) it is hypothesized that stock market crashes are caused by the slow building up of long-range correlations leading to a collapse of the stock market in one critical instant. In fact, Figure 1 illustrates the slow formation of critical crash that happened in the Sri Lankan stock market in 1994. One can observe a staircase-like increase with narrowing width in the index which had lead to a peak on the 1st March 1994 at which the stock price hit the maximum 1378.82. At this point the width of the horizontal line segment is seen vanishingly small. Such a rhythmic behaviour with a shrinking period seems to be a characteristic precursor of crashes similar to the observations made on the Dow Jones Industrial Average for S\&P500 data (Paul and Baschnagel (1999)). (So in contrary to the popular belief, 

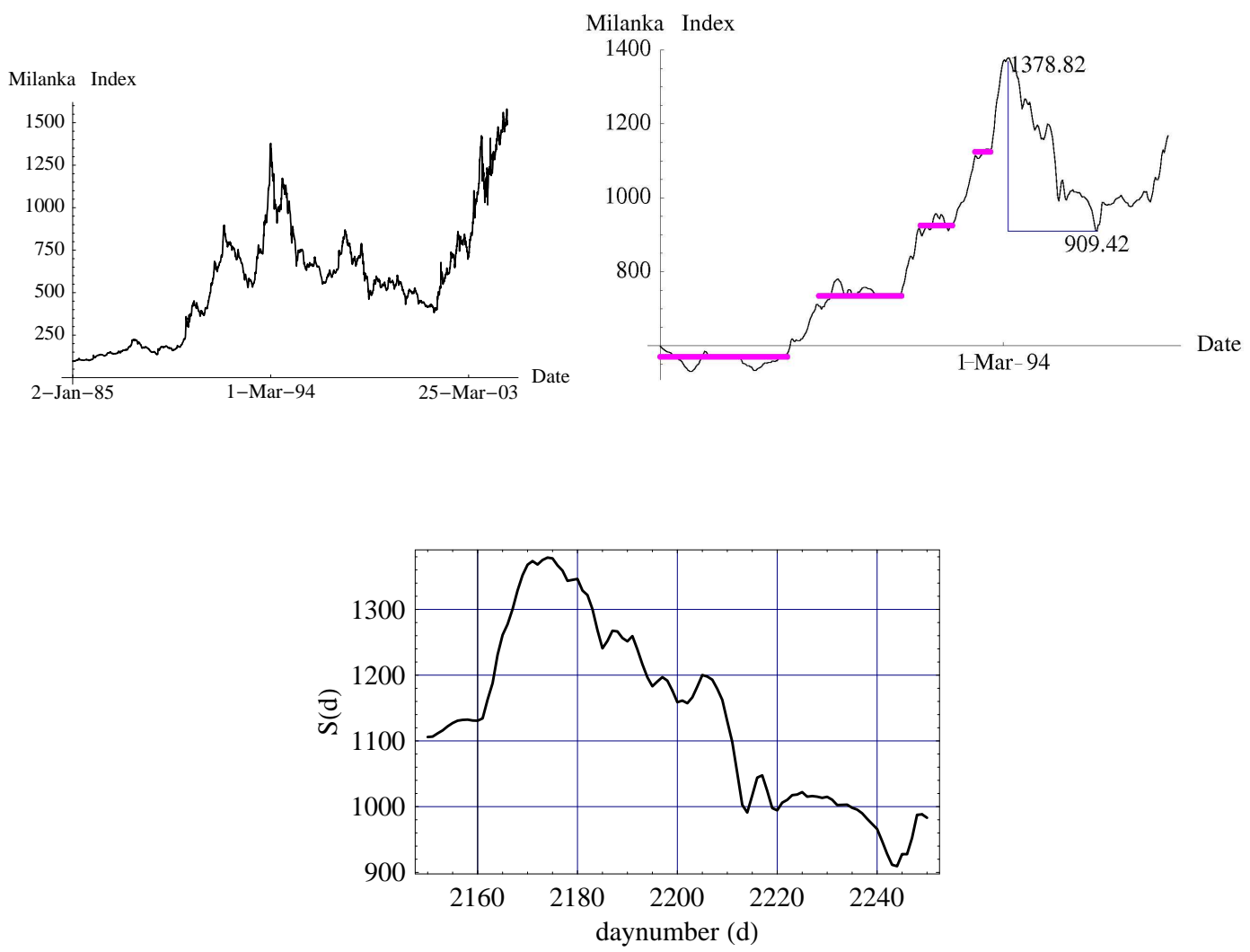

Figure 1 A sudden drop-down of relatively large magnitude (approximately more than 25\%) in a financial index or the stock price is known as a financial crash. Top Left: This data show the evolution of the stock price of the Colombo Stock Exchange over the period 1994 to 2004 . The fall started on the $1^{\text {st }}$ March 1994 when the Milanka Index was at 1378.82 and the local minima in this duration recorded was on 20 June 1994 on which date the Index value was 909.42. Top Right: The evolution of stock price from day 1900 to day 2300. Here we observe the step-like behavior followed by the process (shown in horizontal line segments) whose step size becomes shorter as the market approaches the crash. Bottom: In the model we claim the exact date of the crash as $1^{\text {st }}$ March 1994 corresponding to the day number $t_{C}=2174$ for which the stock price $S\left(t_{C}\right)=L K R 1378.82$. This claim is statistically tested in Section 2.2. ( Source, The Data Disk (2005) - Colombo Stock Exchange.)

ever increasing stock price could be a signal of a possible crash in the future so these situations should be treated with caution).

Statistical fluctuations govern the normal behaviour of the market when supply and demand are well maintained. However, the things start to behave differently 
when the market is in the imminent of a crash. At such times, vast majority of traders spontaneously decide to sell. This unusual cooperativity shakes the liquidity of the market. There is no sufficient demand to defy the exploding supply. The prices drop, and the market falls out of equilibrium (Paul and Baschnagel (1999)). This suggests that crashers are triggered by a spontaneous development of long-range correlations among the traders. Thus, it is important to incorporate the factors that depend on the behaviour of the traders if one is to build-up a model for crashes.

\section{Critical Crashes - Sornette-Johansen Model}

In Johansen et al. (2000) it has been studied the similarities between a phase transition that takes place in a physical system (such as in a magnetic material), and the crashes occur in the financial market. In contrast however, one cannot directly identify a parameter in the financial market which plays the role of a parameter like the temperature in a physical system which drives the system towards a phase transition. It is also argued that such a parameter cannot exist; if it were, the traders in the market would be able to identify it and then shall be able to control it so that a crash wouldn't happen. This suggests that the stimulation for a crash should grow from inside the market. This is a phenomena known as the self-organized criticality (Bak. (1996), Kiyono et al. (2006)) in statistical physics in which it is explained how physical systems undergo phase transitions without any fine-tuning of external control parameters such as temperature or external magnetic field (Paul and Baschnagel (1999)).

In Johansen et al. (2000) it also suggests the imitation as the prime source which drives the market towards a crash. When the prices increase, the traders tend to keep their attention towards the market events. Eventually, some decide to buy with the hope that the trend of price hike will long last. This further increases the prices, which in turn, motivates more traders to speculate. Thus, a speculation bubble is created and this bubble will evolve in time in a breathing like fashion. It is inflated by speculation, but the growth can also stagnate since some traders fear the trend will soon reverse and decide to sell. Even if the number of this kind of traders is small the bubble keeps increasing and eventually reaches a level where many traders assume the price will fall in the future and place sell orders. This fashion or the imitation by the others tend to spread across various levels of the traders in the market. This may trigger a crash with some probability for the bubble to deflate smoothly - without causing a crash.

\subsection{A microscopic model for crashes}

Johansen et al. (2000) introduces a function $h(t)$ called the hazard rate which measures the probability per unit time that a crash will occur in the next time interval $[t, t+\Delta t]$, provided it has not happened before. When the bubble inflates the risk of a crash becomes more likely, thus increases the hazard rate $h(t)$. A possible form for $h(t)$ derived by Sornette et al based on properties of the magnetic 


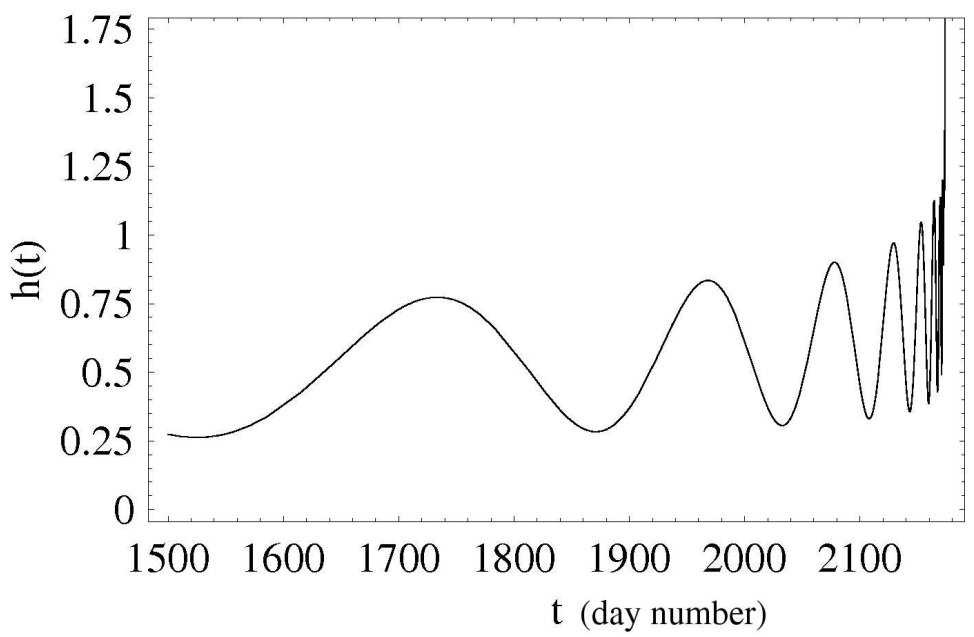

Figure 2 Oscillatory behaviour with the increasing amplitude and shortening period of the function $h(t)$ are the key features of the behaviour of the financial index as it approaches the crash point.

susceptibility of a certain hierarchical diamond lattice proposed by Derrida et al. (1983) is given by

$$
h(t)=\frac{1}{\left(t_{c}-t\right)^{\gamma}}\left[B_{0}+B_{1} \cos \left(\omega \ln \left(t_{c}-t\right)+\psi\right)\right] \quad\left(t \leq t_{c}\right) .
$$

Here, $\gamma$ is a critical exponent which should be universal, i.e., the same for all crashes, $B_{0}$ and $B_{1}$ are constants, and the phase $\psi$ can be related by $\psi=\omega \ln \left(1 / t_{0}\right)$ to a time scale $t_{0}$. This time scale serves to match the equation (1), which should be valid only asymptotically close to the crash, on the data of the price evolution.

The motivation behind having the above ansatz for $h(t)$ is in fact the large increasing trend with oscillations with vanishing periods we observe in the data (see the top-right of the Figure 1). A typical plot of $h(t)$ near the critical point will immediately reveal this (see Figure 2). In Equation (1), we find two major characteristics: Firstly, the term $\frac{1}{\left(t_{c}-t\right)^{\gamma}}$ exhibits a power-law around $t=t_{c}$. Derrida et al. (1983) proved that the susceptibility $\chi$ of the system behaves as

$$
\begin{aligned}
\chi & \approx \operatorname{Re}\left[A_{0}\left(t-t_{c}\right)^{-\gamma}+A_{1}\left(t_{c}-t\right)^{-\gamma+i \omega}+\ldots\right] \\
& \approx A_{1}^{\prime}\left(t_{c}-t\right)^{\gamma} \cos \left[\omega \log \left(t_{c}-t\right)+\psi\right]+\ldots
\end{aligned}
$$

where Re stands for the real part of its argument.

Thus it is reasonable to propose (Johansen et al. (2000)) to consider a form of the equation (2) the hazard rate function. According to (2), we see that in addition 
to the power-law behaviour also is a periodic behaviour which is governed by the trigonometric term.

Sornette et al further obtain the link between $h(t)$ and the actual price evolution. This can also be obtained (Paul and Baschnagel (1999)) by noting the fact that $h(t) \propto \mu(t)$ where $\mu(t)$ is the drift of the stock price process (or the associated geometric Brownian motion). This is obtained as follows: The evolution of the financial index can be decomposed into a deterministic (increasing function of $t$ ) and superimposed stochastic fluctuations. If we neglect the fluctuations and only attempt to model the deterministic increase by geometric Brownian motion, we get the differential equation for the stock price as

$$
d S_{\mathrm{det}}=\mu(t) S_{\mathrm{det}}(t) d t
$$

where $S_{\text {det }}$ stands for the deterministic component of the stock price. This approximation amounts to assuming that the long-term dynamics before the crash is dominated by the drift term with time-dependent $\mu$. Thus it is reasonable to assume

$$
\mu(t)=\kappa h(t)(\kappa>0)
$$

Substituting (5) in (4) and integrating one finds

$$
\ln \frac{S_{\operatorname{det}\left(t_{c}\right)}}{S_{\operatorname{det}}(t)}=\kappa \int_{t}^{t_{c}} d t^{\prime} h\left(t^{\prime}\right)
$$

Inserting (1) into (6) we obtain

$$
\int_{0}^{\tau} d x \frac{1}{x^{\gamma}} \cos (\omega \ln x+\psi)=\frac{1-\gamma}{\omega^{2}+(1-\gamma)^{2} \cos \psi} \tau^{1-\tau} \cos (\omega \ln \tau+\phi) \quad(0<\gamma<1) .
$$

Here, $\tau=t_{c}-t, \tan \varphi=\frac{\omega}{1-\gamma}, \phi=\psi-\varphi,$. Thus we find,

$$
\ln S_{\operatorname{det}(t)}=\ln S_{\mathrm{det}}\left(t_{c}\right)-\left[C_{0} \tau^{\beta}+C_{1} \tau^{\beta} \cos (\omega \ln \tau+\phi)\right]
$$

in which

$$
C_{0}=\frac{\kappa B_{0}}{\beta}, C_{1}=\frac{\kappa B_{1}}{\cos \varphi} \frac{\beta}{\omega^{2}+\beta^{2}}(0<\beta=1-\gamma<1) .
$$

It is essential to have $\gamma \in(0,1)$ as we must have a finite price at $t_{c}$. If $t \approx t_{c}$,

$$
\ln \frac{S_{\mathrm{det}}(t)}{S_{\mathrm{det}}\left(t_{c}\right)} \simeq \frac{S_{\mathrm{det}}(t)}{S_{\mathrm{det}}\left(t_{c}\right)}-1
$$

This solves to,

$$
S_{\text {det }}(t) \simeq S_{\text {det }}\left(t_{c}\right)\left(1-\left[C_{0} \tau^{\beta}+C_{1} \tau^{\beta} \cos (\omega \ln \tau+\phi)\right]\right) .
$$

For further details and the derivation of the above we refer the reader to Paul and Baschnagel (1999). We shall now match already available data to the average 
(a) $t_{C}=2164, M S E=10571.7$. Convergence failed.

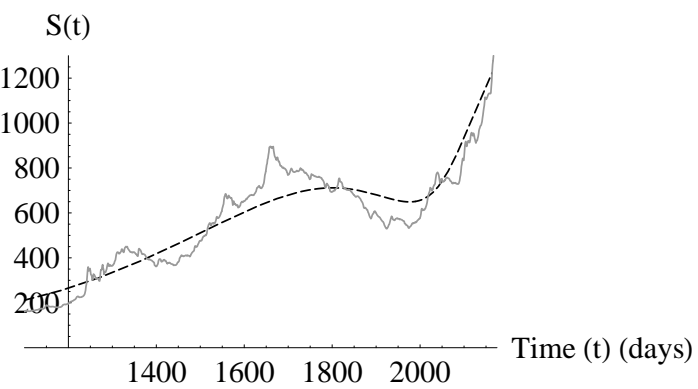

(c) $t_{C}=2174, M S E=3952.99$. Convergence successful.

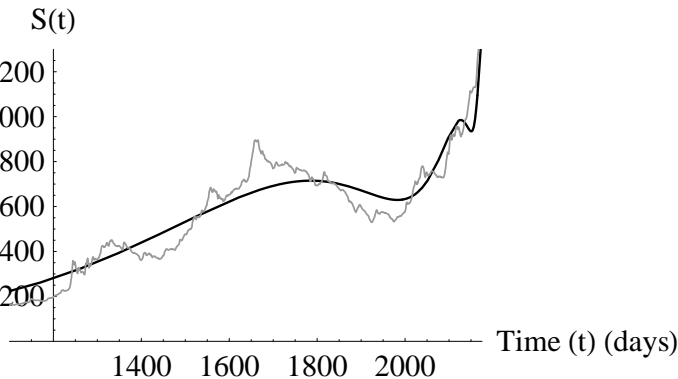

(e) $t_{C}=2180, M S E=4282.94$. Convergence successful.

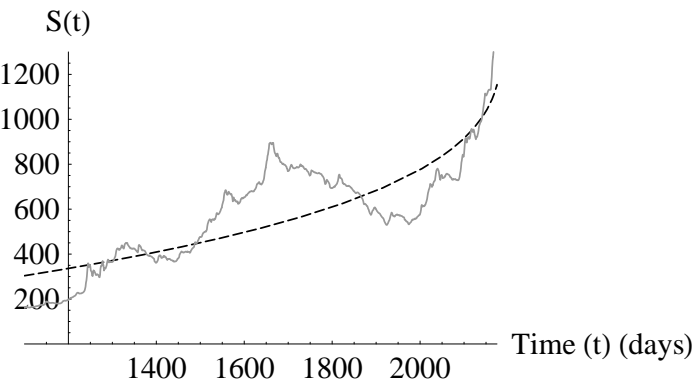

(b) $t_{C}=2171, M S E=3834.67$. Convergence failed.

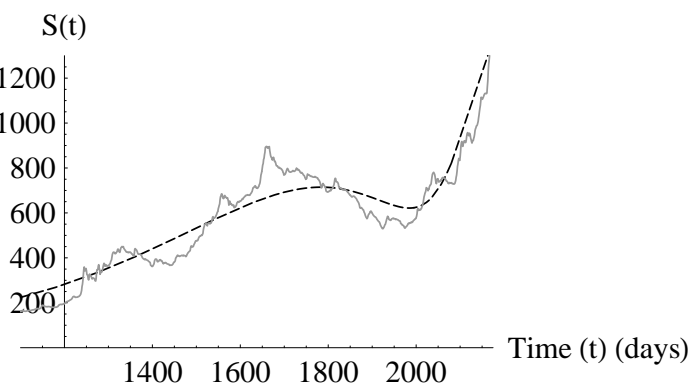

(d) $t_{C}=2177, M S E=10831.4$. Convergence failed.

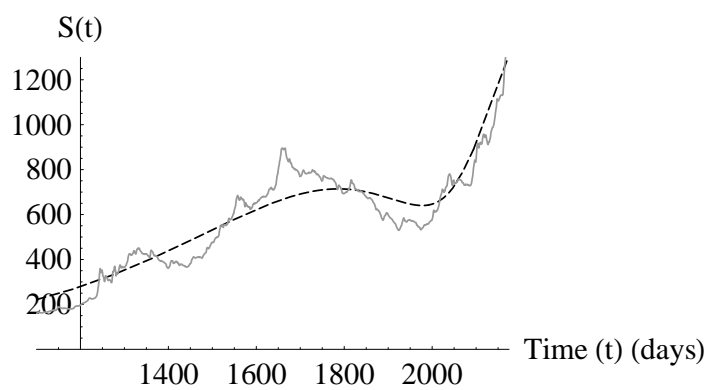

(f) $t_{C}=2183, M S E=10953.4$. Convergence failed.

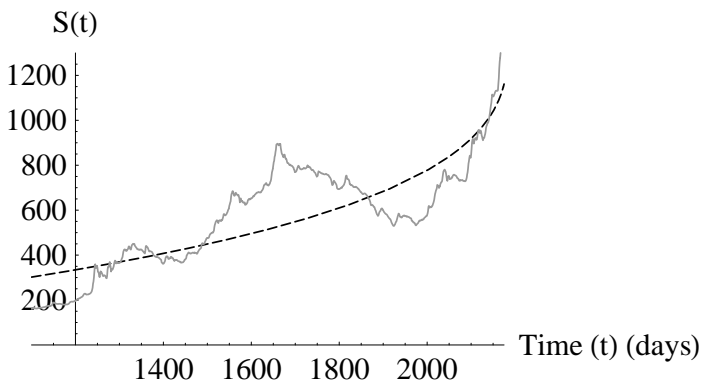

Figure 3 Nonlinear fit for the curve given by the Equation 9. Parameters are estimated for various apriory value for the crash date $t_{C}$ (days) as shown in the bottom of the each plot together with the mean square error values and comments on the convergence of the Levenberg-Marquardt method for nonlinear least-squares available with Mathematica 5.0 Wolfram (2004). The best fit MSE value with successful convergence of the numerical scheme is given in the case (c) which corresponds to the crash date $1^{\text {st }}$ March 1994 we claimed in the caption to Figure 1.

behaviour of the stock price via (9). With this we shall be able to quantify the oscillatory behaviour which is observable prior to a critical crash from the time series data of the stock market. The main difficulty in handling such data using 
latter methods is the divergence of the parameters and indexes in the proximity of the critical time $t_{c}$.

In conclusion, this technique has given us valuable information which are otherwise impossible to infer (for example via statistical time series analysis of data) how probable a critical crash could happen by a given crash date $t_{c}$. On the other hand, the method can be made available to real-time financial information systems to monitor the price evolution and to issue forecasts and warnings to the market.

\subsection{Statistical prediction of the crash date}

In Figure 3 we show the periodic function we obtained by fitting the equation (9) for Milanka data for different a posterior known values for $t_{c}$. The most probable date for the crash is found to be the same as the one we conjectured. This gives the least mean square error with successful convergence of the numerical scheme used as explained in the figure caption. This kind of analysis shall be of extremely useful in decision making made by the investors and management of the market.

\section{How the critical crashes happen ? an explanation from statistical mechanics}

We know that the price of an index (or the stock price as we consider in this section) is a stochastic variable whose value depends on many parameters and variables. Clearly we can plot the stock price $S(t)$ as a function of time, but it is not the time that actually affect the change in $S(t)$ but the activities of agents and other economical and political facts in a society. Needless to say (as we have already discussed in the previous section) not all these factors (or parameters) are possible to be quantified in a model. Nevertheless, we know by our experience that when the agents take decisions at random, the market becomes a highly active place. When the agents tend to speculate and delay taking decisions, the market from the surface seen as a cool place whilst there are ice-berg type activities happening. Here we propose the virtual inverse activity function (VIAF), denoted $\beta$ whose value is inversely proportional to the activity of the market in the sense we discussed in the above for the sole purpose of showing how a critical crash is possible when we model the market via models in statistical physics. In other words,

$$
\begin{aligned}
\beta \text { low } & \Rightarrow \text { market becomes relatively active (or hot) and, } \\
\beta \text { high } & \Rightarrow \text { market becomes less active (or cool) }
\end{aligned}
$$

It is obvious that $\beta$ should also be proportional to the hazard rate function (or be the same as that) as discussed in Section 2.1. (See $p p$. 6 of Johansen et al. (2000)). In the next section we shall show that if we vary $\beta$ from lower values to higher values there is a critical value of $\beta$, say, $\beta_{c}$ beyond which the market enters into a new phase in which the activities of the agents become frozen. This means at the $\beta=\beta_{c}$, the market undergoes a crash or a phase transition as in physical systems. 


\subsection{The model}

Let us consider the evolution of the stock price $S(t)$ over a day. We also assume that the increments and decrements of $S(t)$ over a small time interval (within a day) purely results due to the activities of the agents and that it is these same activities that affect the virtual function $\beta(t)$ to increase or decrease. However, we assume that $\beta(t)$ is fixed during a day (or we consider the average value $\beta(t)$ as the value of the VIAF for the given day. Thus, for a given $\beta$ we let the price evolve in a day over a large number of (say, $n$ ) small time intervals.

We let the up-down behaviour of the price controlled by a Bernoulli random variable $\sigma_{j}= \pm 1$ with probability $p=1 / 2$, during each time interval considered on a day. If there are $n$ time intervals each of duration $\Delta t$, this will resolve a sequence of increments (decrements) $\left\{u_{j}\right\}_{j=1}^{n}$ (See Figure 4).

What we would like to investigate is the evolution of $S(t)$ when $\beta$ increases - or when the market slowly enters into a less active place from a highly active one.

We also assume that $u_{j}$ follows a Log-Normal distribution (in other words this means the $\log$ returns, i.e. $\log u$ follows a Normal (Gaussian) distribution). Indeed, in financial market terminology, the up-down rates $u=\exp (\nu \sqrt{\Delta t})$ where $\nu$ is the volatility, and thus $\log u$ follows a Gaussian distribution scaled by $1 / \sqrt{\Delta t}$ when $\nu$ is Gaussian, i.e. $\nu \sim \mathcal{N}\left(0, \vartheta^{2}\right)$. This means that the change in $S(t)$ is given by $u(t)$.

\subsection{Stochastic Volatility}

By allowing $\nu$ to assume a distribution we consider the situation where the volatility is a stochastic variable rather than a constant. The latter situation is one of the major assumptions in the Black-Sholes theory (Black (1989)).

Thus, the evolution of $u(t)$ is conveniently represented in a binary tree as shown in the Figure 4 . Now a sequence $\left\{u_{j}\right\}_{j=1}^{n}$ corresponds to a path $\omega$, which traverses across all levels of the tree.

Now the problem is equivalent to a self-avoiding random walk along a Cayley tree as it has been treated in (Derrida and Gardner (1985) and, Derrida and Spohn (1988). Going along the same lines as in Buffet et al. (1993) to solve this problem, let us define a path at the top of the tree and of length $|\omega|=n$ as a finite sequence $\left\{\left(j, \omega_{j}\right), 1 \leq j \leq n\right\}$ such that

$$
\omega_{j+1}=2 \omega_{j}-\frac{1}{2}\left(1-\sigma_{j+1}\right),
$$

where $\sigma_{j} \in\{-1,1\}$. The purpose of $\sigma$ 's in the model is to realize a random path in the tree. Moreover, we shall keep the set of values $\left\{u_{i j}\right\}$ unchanged (or quenched) for fixed $\beta$. Attach independent identically distributed random variables $u_{j, k}$ to the bonds of the tree. We assume that the common distribution of $u_{j k}$ obeys $\mathbb{E}[\exp [-\beta u]]<\infty$ for all $\beta \geq 0$. 


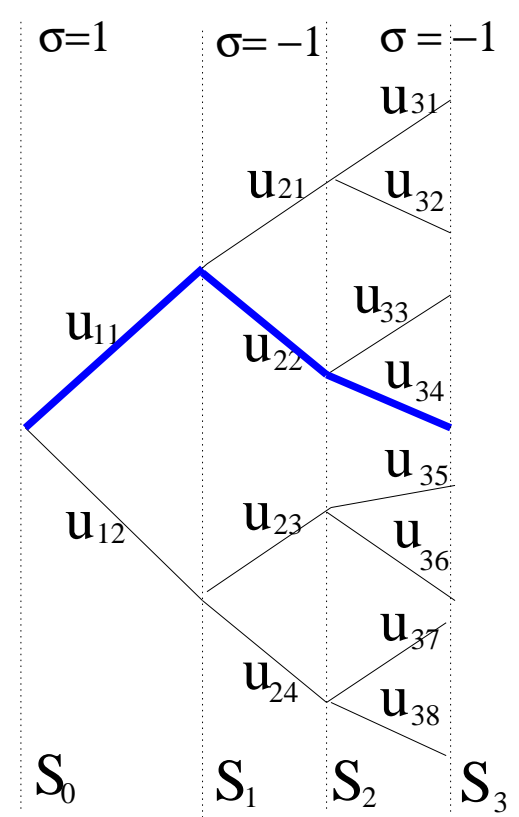

Figure 4 The evolution of the process $u(t)$ over 3 time steps in a day. The thick path shows the price evolution whose up-down movements are characterize by tossing an unbiased coin for which $\sigma= \pm 1$. Thus, if the initial stock price is $S_{0}$ the final stock price $S(t)=S_{0} u_{11} u_{22} u_{34}$. The microscopic description of the system consists of $2^{n}\left(n=3\right.$ here) energy levels with $u_{i j}$ obeying some probability distribution (Ig-normal here).

\subsection{The (free) energy density of the pricing process}

The free energy density of a fluctuating system plays a role similar to the potential energy in mechanics. It is a quantity which relates the entropy and the internal energy of a system in statistical physics. On the other hand the knowledge of free energy density allows one to estimate of all the other thermodynamic quantities such as specific heat, entropy etc. The convergence of free energy density to some finite value for low temperatures implies a phase transition of the system. In the present problem too, we shall proceed to compute the free energy density associated with the process $\left\{u_{j}\right\}$. To do this we force the agents to reduce their activity (or to become highly correlated among themselves) which shall enable us to see what happens to the market as $\beta$ is increased.

For this we first need to have a function to quantify the 'energy' or the Hamiltonian of the system. This we denote by $\mathscr{H}$ and define by

$$
-\mathscr{H}=\log \prod_{j=1}^{n} u_{j},
$$

where $u_{j}=u_{j k}$ at the level $j$ and $k \in\left\{1,2, \ldots, 2^{j}\right\}$. This choice for $\mathscr{H}$ is natural in the financial market context as it is $S_{0} \prod u_{i}$ (where $S_{0}$ is the initial stock price) which determines the final stock price at the end of the evolution. (We identify $S_{0} \prod_{j=1}^{n} u_{j}$ as a possible macroscopic order parameter). 
For fixed $\beta$ the free energy of this process is defined by

$$
f(\beta, \lambda, \gamma)=-\frac{1}{\beta} \lim _{n \rightarrow \infty} \frac{1}{n} \log \mathscr{Z}_{n}(\beta),
$$

where $\beta$ is the average of the VIAF over the total time the market is being observed (over a day in the present case) and $\mathscr{Z}_{n}$ is the partition function defined by

$$
\mathscr{Z}_{n}=\sum_{\omega:|\omega|=n} \exp [-\beta \mathscr{H}]=\sum_{\omega:|\omega|=n} \exp \left[\beta \sum_{j=1}^{n} \log u_{j}\right] .
$$

Notice that $\mathscr{Z}_{n}$ is nothing but the sum of exponentially weighted log returns over all possible paths in the tree.

Let us make the transformation $v_{j}=\ln u_{j}$ in (10) after which the partition function is written,

$$
\mathscr{Z}_{n}=\sum_{\omega:|\omega|=n} \exp \left[-\beta \sum_{j} v_{j}\right],
$$

which is exactly the same partition function obtained in Buffet et al. (1993).

\subsection{Sketch of the derivation of free energy density}

The derivation of the limit in (11) has been done in Buffet et al. (1993) by noting the fact that the normalized partition function

$$
M_{n}(\beta):=\mathscr{Z}_{n}(\beta) /(2 \phi(\beta))^{n}
$$

is a martingale Williams (1991) (i.e. $\mathbb{E}\left[M_{n+1} \mid V^{n}\right]=M_{n}(\beta)$ ), with respect to the filtration $\left\{V^{n}, n \geq 1\right\}$ defined by $V^{n}:=\left\{v_{j, k} ; 1 \leq k \leq 2^{j}, 1 \leq j \leq n\right\}$ - which is the set of all random variables $v_{j, k}$ between the levels 1 and $n$. Here $\phi(\beta):=\mathbb{E}[\exp (-\beta v)]$.

Let us assume for the time being that $M_{n}(\beta)$ is a positive martingale so that it converges to a finite random variable $M_{\infty}(\beta)$ Williams (1991). Moreover, let us also assume that $M_{n}(\beta)<\infty$ with respect to the distribution of $v_{j, k}$. Indeed, these have been proved in Buffet et al. (1993) but for $\beta<\beta_{c}$ for some $\beta_{c}$ which is determined by the parameters of the distribution of $v_{j, k}$. Since

$$
\begin{aligned}
\frac{1}{\beta n} \log \mathscr{Z}_{n}(\beta) & =\frac{1}{\beta n} \log \left[(2 \phi(\beta))^{n} M_{n}(\beta)\right] \\
& =\frac{1}{\beta} \log [2 \phi(\beta)]+\frac{1}{\beta n} \log M_{n}(\beta),
\end{aligned}
$$

we find that

$$
\limsup _{n \rightarrow \infty} \frac{1}{\beta n} \log \mathscr{Z}_{n}(\beta) \leq \frac{1}{\beta} \log [2 \phi(\beta)],
$$


almost surely (a.s.) with respect to the distribution of $v_{j, k}$, since the second term on the right hand side of the equation (14) is vanishing in the limit $n \rightarrow \infty$. Moreover, it is also proved in Buffet et al. (1993) for $\beta<\beta_{c}$ that,

$$
\begin{aligned}
\liminf _{n \rightarrow \infty} \frac{1}{\beta n} \log \mathscr{Z}_{n}(\beta) & \geq \frac{1}{\beta} \log [2 \phi(\beta)] \quad \text { and hence, } \\
\lim _{n \rightarrow \infty} \frac{1}{\beta n} \log \mathscr{Z}_{n}(\beta) & =\frac{1}{\beta} \log [2 \phi(\beta)]:=g_{\vartheta}(\beta) \quad \text { a.s. }
\end{aligned}
$$

However, for $\beta \geq \beta_{c}$ there is no guarantee that the moments of $M_{n}(\beta)$ are finite but since the function $\frac{1}{n \beta} \log \mathscr{Z}_{n}(\beta)$ is decreasing we have for any $\epsilon>0$

$$
\frac{1}{n \beta} \log \mathscr{Z}_{n}(\beta) \leq \frac{1}{n\left(\beta_{c}-\epsilon\right)} \log \mathscr{Z}_{n}\left(\left(\beta_{c}-\epsilon\right)\right)
$$

from which it follows that

$$
\limsup _{n \rightarrow \infty} \frac{1}{n \beta} \log \mathscr{Z}_{n}(\beta) \leq g_{\vartheta}\left(\beta_{c}-\epsilon\right) .
$$

Since $\frac{1}{n \beta} \log \mathscr{Z}_{n}(\beta)$ is convex in $\beta$ it also follows that

$$
\liminf _{n \rightarrow \infty} \frac{1}{n \beta} \log \mathscr{Z}_{n}(\beta) \geq g_{\vartheta}\left(\beta_{c}-\epsilon\right)
$$

and hence

$$
\lim _{n \rightarrow \infty} \frac{1}{n \beta} \log \mathscr{Z}_{n}(\beta)=g_{\vartheta}\left(\beta_{c}\right) \quad \text { if } \beta \geq \beta_{c} .
$$

What we have just illustrated was a sketch of the proof of the following theorem in Buffet et al. (1993):

TheOREM 1. The limit

$$
\lim _{n \rightarrow \infty} \frac{1}{\beta n} \log \mathscr{Z}_{n}(\beta)= \begin{cases}g_{\vartheta}(\beta) & \beta \leq \beta_{c} \\ g_{\vartheta}\left(\beta_{c}\right) & \beta>\beta_{c}\end{cases}
$$

holds almost surely with respect to the distribution of $v_{j, k}$.

REMARK 1. Let us consider an example to determine the $\beta_{c}$ as in the above theorem. Let the random variables $v$ follows a Gaussian distribution with zero mean and variance $\vartheta^{2}$. Then $\beta_{c}=\sqrt{2 \log (2)} / \vartheta$. Figure 5 shows the behaviuor of the function $g_{\vartheta}(\beta)$ plotted for increasing values of $\vartheta$. There is a unique minima for the function and in case $g_{\vartheta}(\beta)$ is strictly decreasing $\beta_{c}$ is considered to be tend to infinity.

The equation (22) shows that the free energy density for an active market (i.e. low $\beta$ ) is governed by the random activities of the agents (as it depends on $\beta$ ), but for $\beta>\beta_{c}$, the internal energy of the system dominates the randomness thus the market behaves as one with frozen activities of the agents (since the free energy is invariant). Figure 6 shows how $f(\beta)$ behaves against $\beta$ as we vary the width $\vartheta$ of the distribution of the price change $u_{j}$. The curves are the plots of the equation (22). 


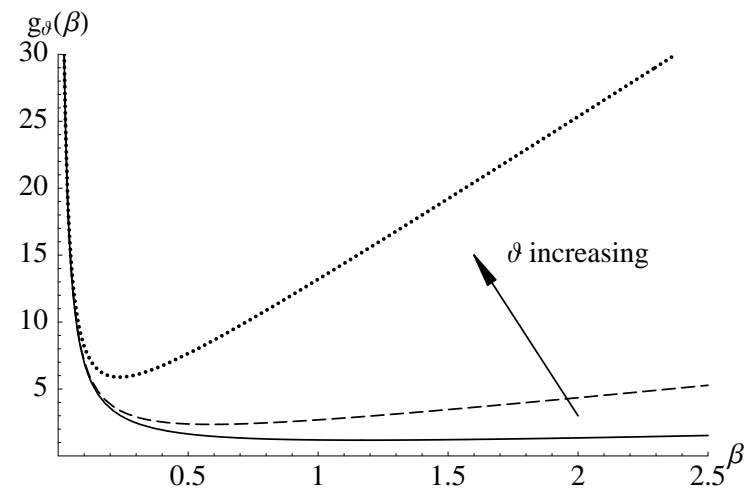

Figure 5 Either there exists $\beta_{c}>0$ such that the function $g_{\vartheta}(\beta)$ is strictly decreasing on $\left(0, \beta_{c}\right)$ and strictly increasing on $\left(\beta_{c}, \infty\right)$ or the function $g_{\vartheta}(\beta)$ is strictly decreasing on $(0, \infty)$. This behaviour is responsible for the apparent discontinuity in the free energy density as it has been proved in Buffet et al. (1993).
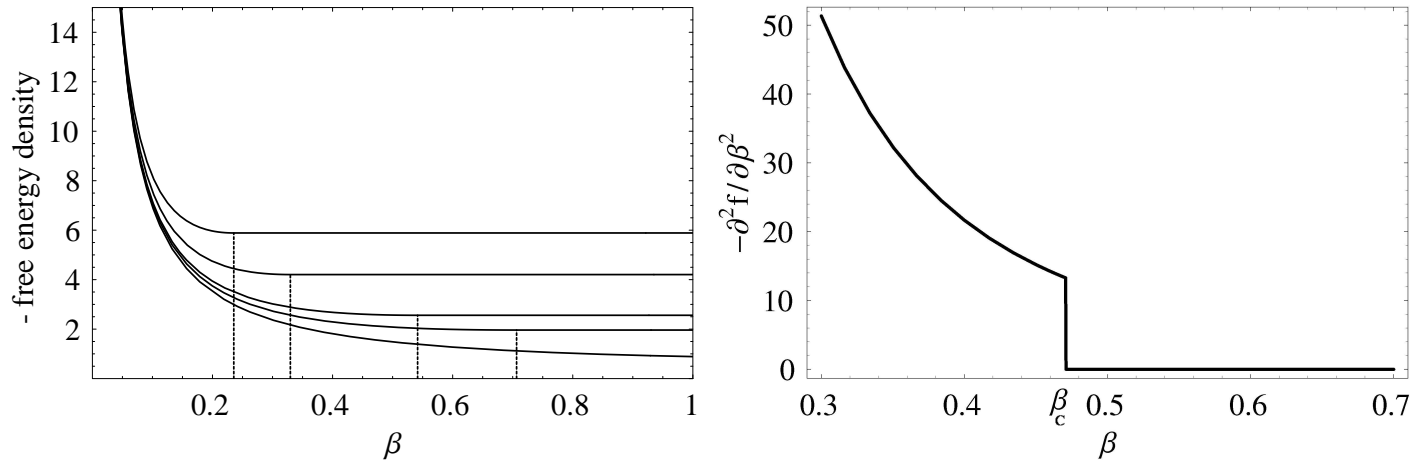

Figure 6 Left: Negative free energy density $-f(\beta)$ as a function of $\beta$ for various values of $\vartheta=$ $.1, .14, .23, .3, .8 . \vartheta$ increases from the top curve to the bottom curve respectively over the above values. Thus when the variation of $u_{j}$ is small, free energy settles at a lower value $\beta$ increases. The vertical line segments indicate the corresponding transition value $\left(\beta_{c}\right)$ for each curve. Right: The second derivative of $f(\beta)$ has a discontinuity at $\beta=\beta_{c}$. This implies a crash (phase transition) at $\beta_{c}$ and moreover, we identify this as a second order phase transition in the statistical physics terminology.

By varying $\beta$ what we illustrate here is how the free energy density of the market behaves up-to the day on which the crash occurs. As it is shown in Figure 6, we notice that the free energy is unchanged for $\beta>\beta_{c}$, which in the context of financial market means that, the long range correlations among the agents dominates in this regime over the random activities of the agents.

Another characteristic of physical systems that exhibit phase transitions is the discontinuities of thermodynamic quantities such as density, pressure or free energy density. In the present case we see that the second derivative of the free energy has 
a discontinuity at $\beta=\beta_{c}$ : Indeed, we find

$$
\frac{\partial^{2}}{\partial \beta^{2}} f(\beta)= \begin{cases}2 \log 2 / \beta & \beta \leq \beta_{c} \\ 0 & \beta>\beta_{c} .\end{cases}
$$

The above discontinuity characterizes a so called second order phase transition in statistical mehcanics.

\section{Conclusions}

As far as the authors are concerned, the Sri Lankan stock market has never been studied for critical crashes using the methods discussed in this paper or by any other means. As such, we believe that these reporting will provide with some valuable insight to the market specially in carefully monitoring the market for precursors that sign a possible crash. Using these the decision makers can have an a-priory estimate of the possible crash date - but as Johansen et al. (2000) points out the date of the actual crash is purely random. There is no requirement that this date be coincide with the date estimates using the above method.

The results we obtained in Section 3 exhibit how a phase transition or a critical crash takes place in the market when we control a virtual parameter, namely $\beta$. It would be worthwhile to see how $\beta$ is related to the hazard rate function introduced in Section 2.1. Our work in this regards is purely pedagogical and illustrative as in practice it is not possible to find a control parameter such as $\beta$ for markets (Paul and Baschnagel (1999)). Nevertheless, the markets are self-evolved due to the internal activities of the interacting agents towards crashes in which situations one can see the inverse activity function $\beta$ increase. So there is no harm of varying $\beta$ to see how the market will respond to these changes which we have quantified by the free energy density function $f(\beta)$. The discontinuity in the second derivative of the free energy function means the market entering into a new regime.This, when translated into the pricing process would imply that the trend of the pricing process could alter. Indeed, one can see just after the crash-point the overall trend of the pricing process starts to decrease.

The results clearly show the crashes can be explained and they are not unusual phenomena in the market from the statistical mechanics point of view.

\section{Acknowledgments}

The authors wish to thank the reviewers of the paper for their valuable comments and also to the Department of Mathematics, University of Ruhuna and the Colombo Stock Exchange - Matara Office, Sri Lanka, for their support given in this work.

\section{References}

Bak P. 1996. How Nature Works: The Science of Self-Organized Criticality. SpringerCopernicus, New York.

Black, F. 1989. How we came up with the option formula. J. Portfolio management 154. 
Buffet, E., A. Patrick, J. Pulé. 1993. J. Phys. A 261823.

Derrida, B. 1980. Random-Energy Model: Limit of a Family of Disordered Models. Phys. Rev. Lett. 4579.

Derrida, B. 1981. An exactly solvable model of disordered systems. Phys. Review B24 2613.

Derrida, B. 1985. J. Physique. Lett 46 L401.

Derrida, B. 1997. From random walks to spin glasses. Physica D 186-198.

Derrida, B., E. Gardner. 1985. Solution of the generalised random energy model. J. Phys C $192253-2274$.

Derrida, B., H. Spohn. 1988. Polymers on disordered trees, spin glasses, and travelling waves. Jn. Stat. Physics 51 817-840.

Derrida B., De Seze L., Itzykson C. 1983. Fractal structure of zeros in heirachical models. Jnl. of Statisical Physics 33559.

Dorlas, Tenuis C. 1999. Statistical Mechanics - Fundamental and Model Solutions. Institute of Physics Publishing London.

Ken Kiyono, Zhigniew R. Struzik, and Yoshiharu Yamamomoto 2006. Criticality and phase transition in stock-price fluctuations. Phys. Rev. Lett. 96 068701-1-068701-4.

Johansen A., Sornette D. 1998. Critical crashes are outliers. Eur. Phys. J. B1 141.

Paul, Wolfgang, Jörg Baschnagel. 1999. Stochastic Processes From Physics to Finance. Springer.

Schwabl, Franz. 2002. Statistical Mechanics. Springer-Verlag.

Sornette D., Bouchand JP., Johansen A. 1996. Stock market crashes, precurses and replicas. J. Phys I France 6 167-175.

Johansen A., Ledoit O., and Sornette D. 2000 Crashes as Critical Points Int. J. Theor. Applied Finance 3219.

Williams, D. 1991. Probability with Martingales. Cambridge University Press.

Wolfram, S. 2004. http://www.wolfram.com.

Yeomans, JM. 1992. Statistical Mechanics of Phase Transistions. Oxford University Press. 